\title{
Jerarquización del Reemplazo de Equipos Productivos de Acuerdo a su Nivel de Cumplimiento de los Objetivos de la Empresa
}

\author{
Fernando F. Espinosa, Gonzalo E. Salinas, Pedro P. Leiva \\ Facultad de Ingeniería, Universidad de Talca, 2 Norte 685, Talca-Chile \\ (e-mail: fespinos@utalca.cl; gsalinas@utalca.cl) \\ Recibido Sep. 29, 2010; Aceptado Nov. 22, 2010; Versión Final recibida Dic. 22, 2010
}

\begin{abstract}
Resumen
Se presenta la implementación computacional de un modelo de ayuda para el administrador del mantenimiento industrial, quien debe decidir sobre la jerarquización del reemplazo de equipos productivos. El sistema considera el nivel de cumplimiento de los requisitos de los nuevos productos que la empresa desea producir para satisfacer la evolución de estos en el mercado. El modelo jerarquiza el orden del reemplazo del equipo basado en su criticidad, grado de adaptabilidad a la evolución del producto y el costo de un equipo nuevo. Todos los antecedentes que son ingresados en las planillas de cálculo reflejan la apreciación que el equipo de mantenimiento tiene sobre su entorno. Los resultados son analizados y presentados gráficamente.
\end{abstract}

Palabras claves: mantenimiento industrial, reemplazo de equipos, criticidad, jerarquización.

\section{Hierarchical Structure of the Replacement of Productive Equipment according to the Fulfillment of the Company's Objectives}

\begin{abstract}
In this paper the computational implementation of a model for the administrator of industrial maintenance, the person who must decide about the priority in which the productive equipments in a company should be replaced. The system takes into account the level of importance in the evolution of new products that the company wants to produce and to satisfy the ones already in the market. The program gives priority to the replacement of equipment based on its impact on productivity, the level of adaptability with the evolution of the product and the replacement cost of the new equipment. All of the data introduced on the spreadsheet reflect the appreciation that of the piece of equipment has on its surrounding. The results are analyzed and graphically presented.
\end{abstract}




\section{INTRODUCCIÓN}

Con las tendencias actuales de producción Just-in-Time (JIT), ajustada (Lean Production) y producción flexible y ágil, es vital que la gestión del mantenimiento esté integrada con las estrategias de la empresa para asegurar la disponibilidad del equipamiento, la calidad del producto, la entrega a tiempo, el precio competitivo y el respeto a las normas de seguridad (Riis et al. 1996). Las decisiones estratégicas involucran evaluar detalladamente la pertinencia de invertir recursos financieros en actividades para innovar la forma de realizar el mantenimiento, conjuntamente con el nivel tecnológico de los equipos productivos, con la finalidad de entregar a la empresa un real apoyo para hacerla más competitiva (Carneiro, 2006; Kumar et al., 2009). En efecto, factores tales como alta confiabilidad, mantenabilidad, disponibilidad y seguridad, además del bajo costo de mantenimiento, alta calidad de los productos elaborados, depreciación adecuada de los equipamientos, pueden dar un impacto fundamental a la empresa para ser altamente competitiva (Shyjith, 2009).

El producto o servicio que una empresa ofrece al mercado consumidor es el reflejo de su nivel o capacidad tecnológica, pero este producto debe poseer ciertas características de calidad para el uso previsto, tener un tiempo determinado para llegar a su destino final y ser producido en un volumen que es necesario satisfacer. Para todo esto la función mantenimiento tiene un papel muy importante: es una de las responsables directa para que estos requisitos se cumplan. Por un lado se tienen los requisitos del mercado y por otro las capacidades actuales de la función mantenimiento. Usando una metodología estructurada se pueden determinar todas las debilidades y fortalezas (Bhuiyan y Baghel, 2005), lo que permite definir cuáles son las acciones más apropiadas para mejorar la calidad del servicio de mantenimiento $y$, por consiguiente la competitividad de la empresa.

En este trabajo se explora la condición de los equipamientos para atender a los objetivos de la empresa, conjuntamente con los servicios relacionados con la disponibilidad y sustentabilidad total, a saber: mantenimiento, operación, abastecimiento (logística) y capacidad administrativa. La metodología utilizada consistió en fijar los parámetros utilizados por los administradores del mantenimiento en la toma de decisiones para este tipo de problema, y en base a estos parámetros definir indicadores y criterios para la jerarquización de equipos que pueden ser reemplazados.

\section{EVALUACIÓN DEL NIVEL DE ATENDIMIENTO}

Una vez identificados los equipos y procesos productivos involucrados en la evaluación, en el siguiente paso, el nivel de atendimiento de los equipamientos, permite relacionar recursos técnicos y recursos humanos (servicios) con los requisitos relativos a: evolución del producto en el mercado, resultados económicos, entre otros. La ponderación para la valoración del nivel sigue el siguiente criterio:

4: Atendimiento excelente: El equipamiento puede responder a los requisitos sin introducir modificaciones tecnológicas o de arquitectura.

3: Atendimiento bueno: El equipamiento puede responder bien a los requisitos de buena forma, sólo es necesario realizar adecuaciones pequeñas que no comprometen la capacidad de producción.

2: Atendimiento con dificultad: Para alcanzar los requisitos es necesario realizar una inversión en el equipamiento para adquirir la tecnología adecuada para fabricar el nuevo producto.

1: No atiende los requisitos: El equipamiento está obsoleto o para realizar las modificaciones necesarias se tiene que incurrir en un costo muy alto, lo que torna inviable el proyecto.

Para los servicios que están relacionados con el funcionamiento de los equipamientos, como el mantenimiento, operación, logística y administración, la valoración se refiere a la capacidad de respuesta, o sea, si los servicios tienen la capacidad de responder a los requerimientos necesarios, en frente de modificaciones en las condiciones del entorno. En el caso del mantenimiento, si esta función puede implementar las nuevas tecnologías y mantenerlas operando. Así para operaciones, si el personal está capacitado para integrarse a los cambios 
tecnológicos; para el abastecimiento, si puede adquirir la tecnología y sustentarla con un flujo continuo de piezas y finalmente, para la administración, si posee la rapidez de respuesta suficiente para no disminuir o detener el ritmo de adquisición de la tecnología y de las piezas. La pantalla de captura de datos se muestra en la Fig. 1.

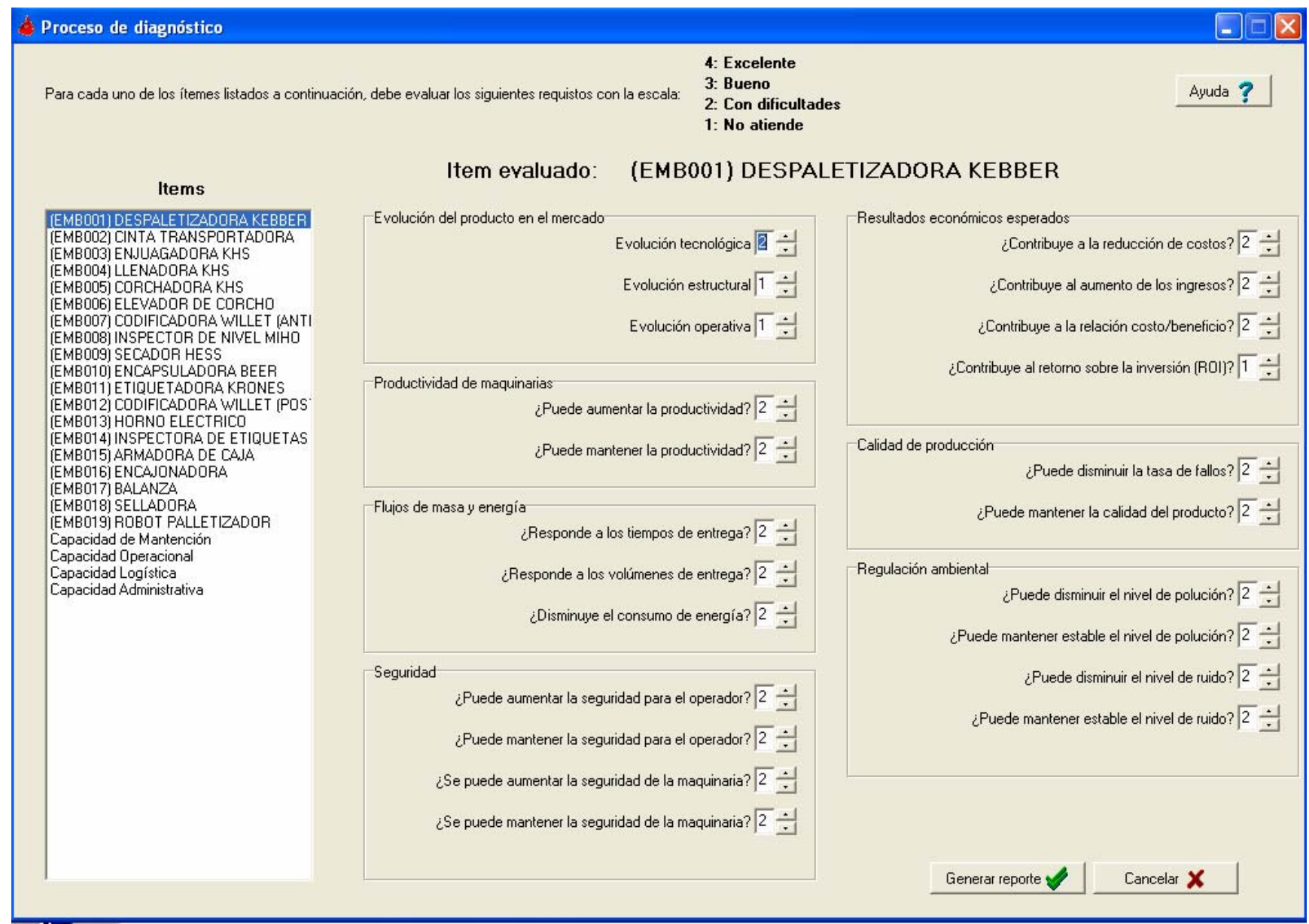

Fig. 1 Parte de la pantalla con datos para evaluar el nivel de atendimiento de los requerimientos

Cada requisito incluido en este módulo es evaluado por un proceso de multicriterio, cuya graduación es definida, para cada punto del análisis, según la siguiente orientación:

Evolución del producto en el mercado. Atiende la evolución tecnológica: si el equipamiento está en condiciones de procesar e integrar nuevas tecnologías en el producto; atiende la evolución estructural: si el equipamiento está en condiciones de procesar e integrar nuevos materiales y arquitecturas en el producto; $y$, atiende la evolución operativa: si el equipamiento está en condiciones de procesar e integrar nuevas formas de operar o manipular el producto.

Resultados económicos esperados. Contribuye a la reducción de costos: la función mantenimiento está en condiciones de reducir sus costos mejorando la gestión de los recursos y si el equipamiento permite un proceso productivo más eficiente; contribuye al aumento del facturamiento: si el equipamiento puede aumentar la calidad del producto elaborado y consigue así un mejor margen de contribución; contribuye al retorno de la inversión: si realizando un efectivo mantenimiento se consigue disminuir la pérdida de valor de los equipamientos en el tiempo; y, contribuye a la relación costo/beneficio: si la función mantenimiento contribuye con el resultado global de la empresa, mediante la disminución de costos operacionales.

Productividad de los equipamientos. Aumento de la productividad: si existe la capacidad de la función mantenimiento y de los equipamientos para aumentar la productividad vía mayor automatización o re-proyecto de los mismos; y, mantener la productividad: si existe la capacidad 
de la función mantenimiento y de los equipos para mantener los niveles actuales de productividad evitando el deterioro acelerado.

Calidad de la producción. Disminución de la tasa de rechazo: si existe la capacidad de la función mantenimiento y de los equipamientos para introducir mejoramientos tecnológicos que incrementen los niveles actuales de calidad del producto; y, mantener la tasa de rechazo: si existe la capacidad de la función mantenimiento y de los equipamientos para mantener los niveles actuales de calidad del producto.

Flujos de masa y energía. Atiende los tiempos de entrega: la función mantenimiento y los equipamientos deben mantener la disponibilidad de acuerdo con las exigencias de los tiempos de entrega de los productos; atiende los volúmenes de entrega: los equipos productivos deben garantizar la disponibilidad y la capacidad de producción conforme a las exigencias de los volúmenes de entrega de los productos, a partir de la función mantenimiento en el sentido de garantizar la tasa de productividad; y, atiende los consumos de energía: si la eficiencia de los equipamientos es alta para no consumir energía extra en su funcionamiento.

Reglamentación ambiental. Disminuye los niveles de polución: o sea, disminuir todo material que al final pueda traducir en contaminación, como: caída de lubricante, productos rechazados, piezas de repuestos usadas y acumuladas, virutas del procesos de fabricación, etc.; mantener los niveles de polución: evitar que la contaminación aumente, o sea, mantener los contaminantes en el nivel actual; disminuye los niveles de vibraciones: si existe un plan o capacidad de disminución de los niveles de vibraciones para dar al trabajador mayor confort y para proteger el ambiente de trabajo; y, mantener los niveles de vibraciones: evitar que los niveles de ruido aumenten y lleguen a ser nocivos para la salud del operario y para el ambiente de trabajo.

Requisitos de seguridad. Aumentar la seguridad para el operador: si existe la posibilidad de implementar acciones para evitar la ocurrencia de accidentes a los colaboradores en la operación de los equipamientos; mantener la seguridad para el operador: implementar un plan de mantenimiento para tener en excelente estado de barreras actuales y así evitar la ocurrencia de accidentes; aumentar seguridad del equipamiento: si existe la capacidad para implementar un plan de acciones de mantenimiento y proyecto para incrementar el estado de auto-protección de las máquinas; y, mantener la seguridad del equipamiento: implementar un plan de mantenimiento para tener en excelente condiciones el estado actual de auto-protección de la maquinaria.

El conjunto de preguntas definido para evaluar el nivel de atendimiento de los requisitos que se muestra en la Fig. 1, son calificados conforme a la visión del equipo de personas que pertenece a la organización del mantenimiento.

La lista de condiciones levantadas es la más simple que la gerencia de una empresa podría someter a la función mantenimiento. Esta lista no es exhaustiva aún siendo relativamente amplia pudiendo, en el caso de haber alguna condición particular de una empresa, ser agregado o substituido por otro que sea de real importancia. El objetivo de este paso es tener una visión bastante clara del nivel de atendimiento de los equipamientos, a esos requisitos, como también el nivel de los servicios que la empresa tiene y que están relacionados con el parque de máquinas.

Como salida de esta evaluación, se tiene entre otros, los gráficos mostrados en las Fig. 2 y 3 , donde se presentan los antecedentes para que el equipo de mantenimiento realice un primer análisis: ¿el nivel de atendimiento de los requisitos de producción es el adecuado para enfrentar los desafíos de la empresa? Es importante esta decisión porque los objetivos de la función mantenimiento deben ser congruentes con los objetivos del negocio de la empresa (Crespo et al., 2009). Los resultados en porcentaje son expresados en función de la ponderación 3 , o superior, para el atendimiento en cada condición evaluada. Se entrega además un informe con observaciones, que son almacenadas en su base de datos por el facilitador a medidas que este sistema es aplicado sucesivamente, las cuales se seleccionan conforme a los resultados arrojados por el análisis de los datos entregados por el administrador del mantenimiento encuestado. 
Los resultados basados en los antecedentes son formateados en gráficos de barras, ya sea para los equipamientos o para los requisitos expresados en forma de preguntas. Esta forma de presentación es bastante conveniente para la toma de decisión en conjunto, debido a la fácil visualización de todos los aspectos evaluados. En este punto de la aplicación de la metodología, el analista ya tiene una visión más profunda de las capacidades de sus recursos, ya que se vio en la necesidad de compatibilizar la información actual con una prospección de futuro, o sea, lo que la empresa espera obtener de sus recursos tanto físicos como humanos.

\section{ANÁLISIS DE LA CRITICIDAD DE LOS EQUIPAMIENTOS}

El análisis de la criticidad de los equipamientos en este proceso tiene dos objetivos: el primero define el tamaño del proyecto para la implementación de las estrategias del mantenimiento, y el segundo ayuda a establecer un orden jerárquico para el uso de los recursos, ya que de esta forma se concentran los esfuerzos en aquellos equipos que impactarán más fuerte en la efectividad de la línea de producción y del mantenimiento y así garantizar la innovación que se introducirá.

La definición de la extensión de la aplicación de la innovación es importante porque está relacionada, directamente con la capacidad y los recursos que la organización dispone. Si la experiencia en proyectos innovadores es relativamente reciente, no es conveniente abarcar todos los equipamientos simultáneamente ya que la probabilidad de fracaso puede aumentar (Chan et al. 2005; Rentes, 2000; Coetzee, 1999). Las causas que llevarían al fracaso de la aplicación están centradas, básicamente, en la falta de conocimiento y en la falta de la información necesaria para lo que se quiere innovar o cambiar. Esto puede llevar a repetir lo que ya se conoce, sin saber lo que hay que cambiar.

También es conveniente definir el tamaño del cambio teniendo en vista la condición económica. Si los recursos son limitados, es mejor asegurar una buena implementación en lo que es prioritario, con mayor repercusión para la producción y para el aprendizaje y para seguir adelante con la innovación, y para tal efecto uno de los antecedentes solicitados es el costo del equipamiento nuevo ya que pasa a formar parte de los indicadores que apoyan la toma de decisiones. El modelo usado para definir la criticidad del equipamiento está basado en cuatro aspectos: velocidad de manifestación de la falla, seguridad del operador y ambiente, pérdidas de producción y costos de reparación (Suárez et al., 2003). Dependiendo de la naturaleza de la empresa, el administrador tiene la facilidad de ponderar cada uno de estos factores y, así reflejar mejor sus contingencias y prioridades (Fig. 4).
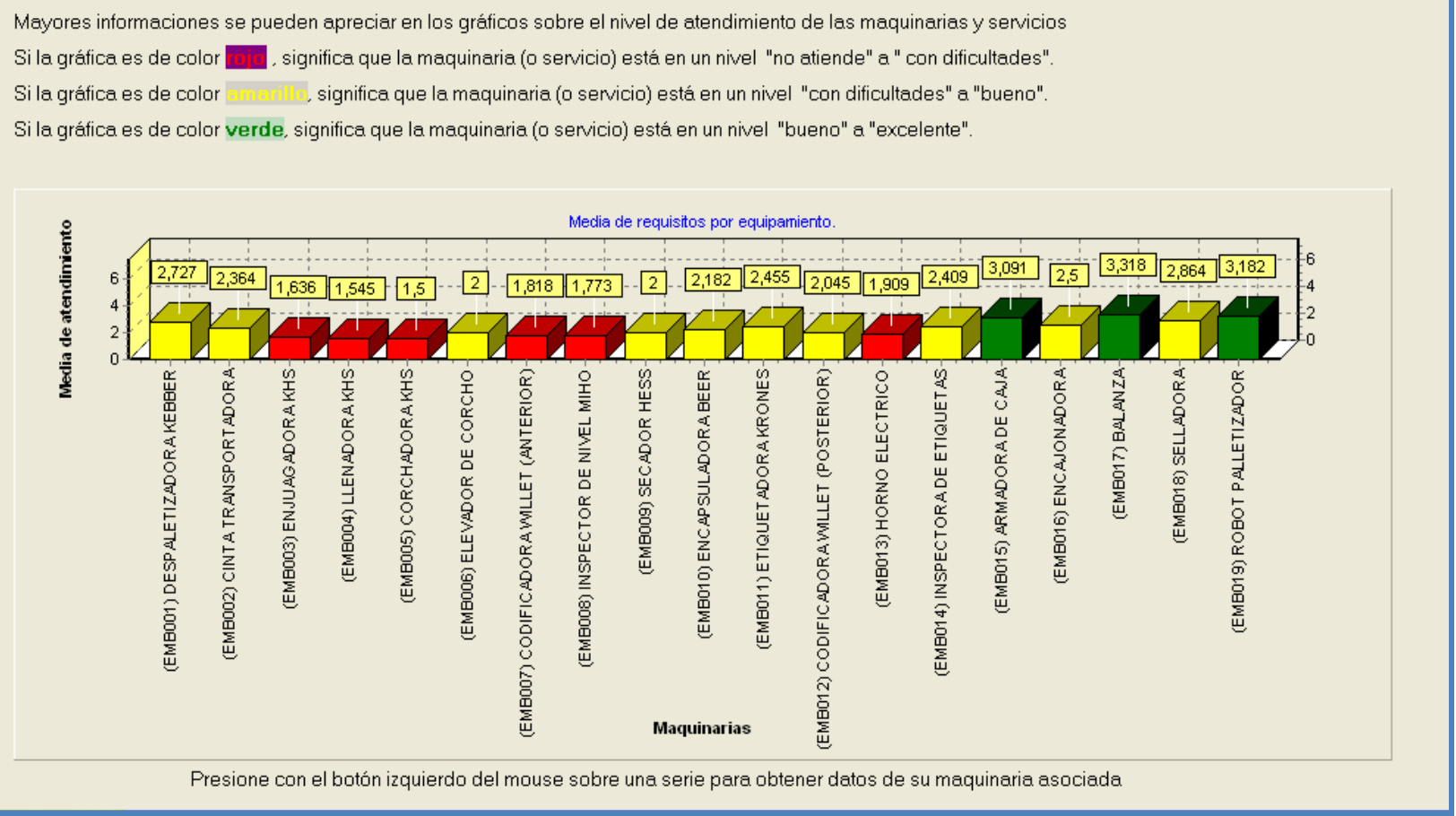

Fig. 2 Gráfico de la media del atendimiento a los requisitos de los equipamientos 


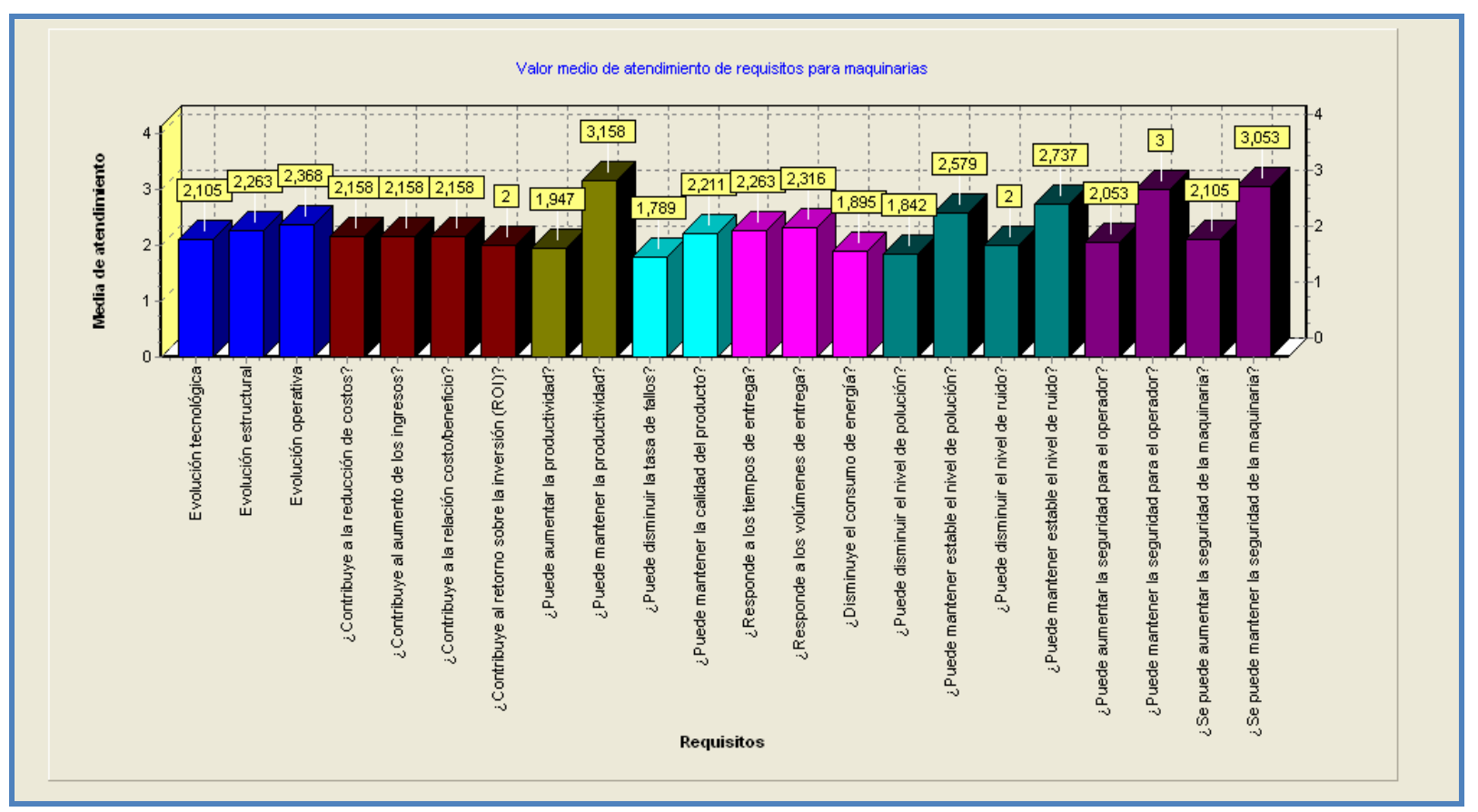

Fig. 3 Gráfico de la media del atendimiento a los requisitos

El criterio para definir la criticidad es el siguiente: si el valor del parámetro de criticidad es mayor o igual a 87 la máquina es declarada "Crítica", significa que tres de los aspectos contenidos en la evaluación son de alto impacto y uno de ellos es de mediano impacto, o todos son de alto impacto. Que sea "Semi-crítico" (valor entre 87 e 50) significa que todos los aspectos son de mediano impacto o contiene sólo una condición de alto impacto. Para las restantes condiciones la clasificación es "No crítico". Cada ítem de este módulo está integrado por: factor de velocidad de manifestación de la falla, factor de seguridad del personal y del ambiente, factor de costos de parada de producción y factor de costos de reparación

Factor de velocidad de manifestación de la falla

Período P-F (Potencial Failure - Functional Failure): es el tiempo que puede transcurrir entre el momento en que se detecta una falla potencial y el momento en que esta se transforma en falla funcional. La escala de valoración es: muy corto, no da tiempo para parar el equipamiento; corto, es posible parar el equipamiento; suficiente, es posible programar la intervención.

\section{Factor de seguridad del personal y del ambiente}

Descripción: el foco es evaluar las consecuencias que la falla podría ocasionar sobre las personas y su impacto sobre el ambiente. La escala es: sin consecuencias; efecto temporal sobre las personas, no afecta el ambiente; efecto temporal sobre las personas y el ambiente; efecto irreversible sobre las personas; efecto irreversible sobre las personas y el ambiente.

\section{Factor de costos de parada de producción}

Criterio: permite establecer criterios para la categorización de los equipamientos conforme a las consecuencias sobre el proceso de producción y satisfacción de la demanda. La escala es: no implica demora en la entrega; implica demora leve en la entrega; implica demora y pérdida de clientes.

\section{Factor de costos de reparación}

Clasificación de acuerdo con Pareto: permite determinar criterios de clasificación de las fallas de acuerdo con los costos directos de reparación. La escala usada es: clasificación A: equipamiento que pertenece al grupo responsable por el $80 \%$ del total de los costos directos de reparación; clasificación B: equipamiento que pertenece al grupo responsable por el $15 \%$ del total de los costos directos de reparación; clasificación C: equipamiento que pertenece al grupo correspondiente al $5 \%$ del total de los costos directos de reparación. 


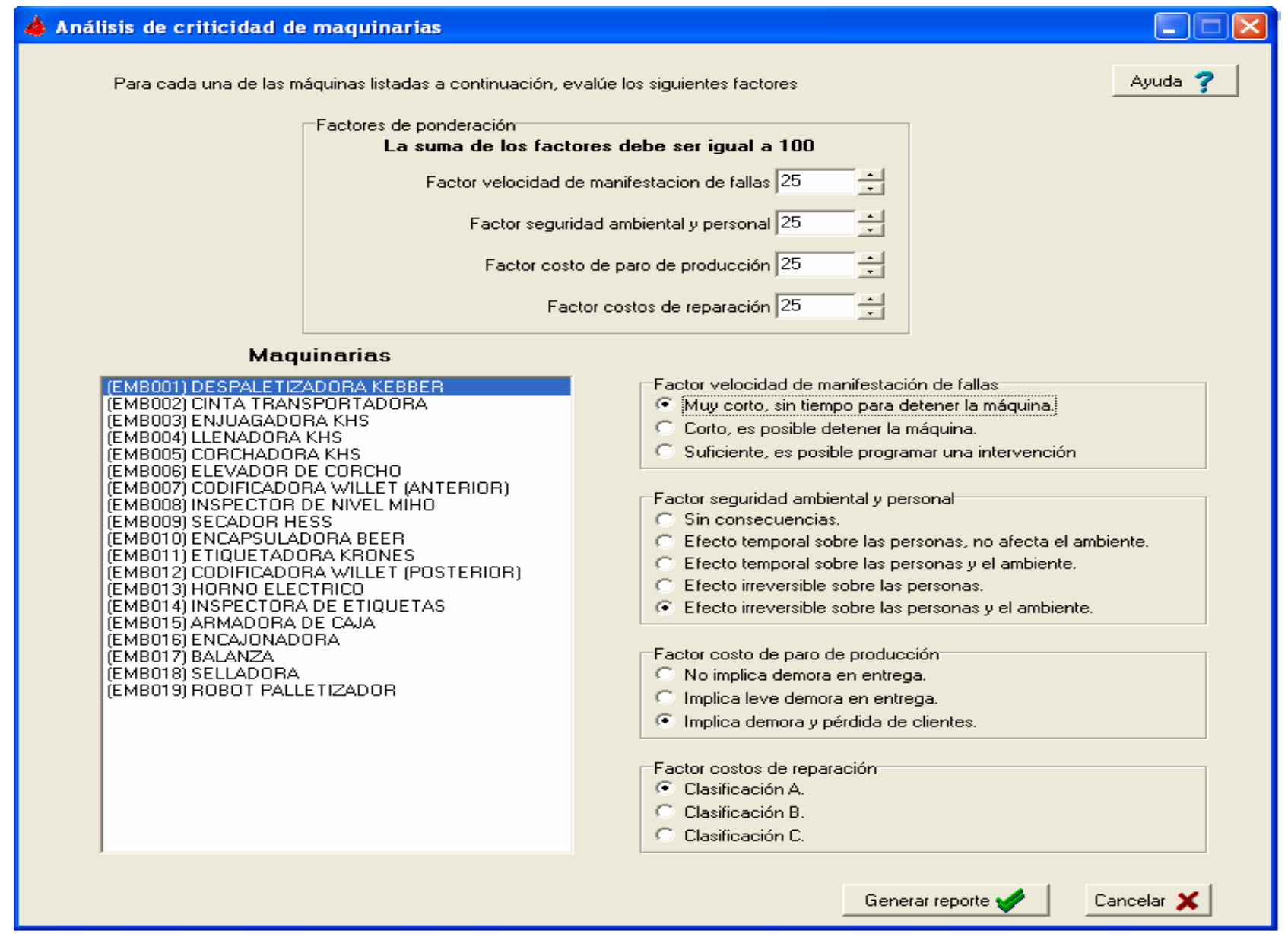

Fig. 4 Planilla para introducción de datos para el análisis de criticidad

\section{JERARQUIZACIÓN DE LA INVERSIÓN EN EL PARQUE DE EQUIPAMIENTOS}

Para solucionar el problema de la obsolescencia o inadecuación de algunos equipamientos de la línea de producción, generalmente la solución más inmediata es adquirir la máquina o tecnología e instalarla. Pero el problema no es tan simple como parece: tendrá que asegurarse de que la organización (producción y mantenimiento específicamente) puede absorber la tecnología (Chen y Small, 1996) y que la línea de producción, por otro lado, no sea perjudicada por la inserción de un nuevo componente (generación de inventarios intermedios o elevación de costos de producción por sub-utilización).

El impacto de la introducción de un nuevo equipamiento en la línea de producción no solamente se refleja en la célula de donde hará parte integrante, sino que también repercute en todos los procesos que son realizados en la línea de producción. El proceso para la captura de esta realidad se muestra en la Fig. 5, donde se muestran los criterios de valoración para cada situación.

El mejoramiento de un proceso lleva a adecuar los demás equipamientos o procesos, para que de esta forma la ganancia en una célula no se convierta en pérdidas en las otras. El problema se amplia cuando los puntos que necesitan mejoramientos son varios y el presupuesto es limitado. Tendrá entonces que tomar decisiones sobre las prioridades de inversión sobre los equipamientos.

El procedimiento mostrado en la Fig. 6 usa un conjunto de indicadores, apoyados en los antecedentes obtenidos de la aplicación de la metodología y otros que son entregados por el analista. Los antecedentes obtenidos de la aplicación de la metodología son la criticidad de cada equipamiento y su nivel actual de adecuación a las metas impuestas para el equipamiento que está sujeto al estudio del mantenimiento. El administrador debe introducir el costo de cada equipamiento, el impacto del equipamiento nuevo sobre los restantes de la línea (valor positivo o negativo) y la apreciación de la capacidad de adecuación de las personas e infra-estructura a la nueva tecnología. 


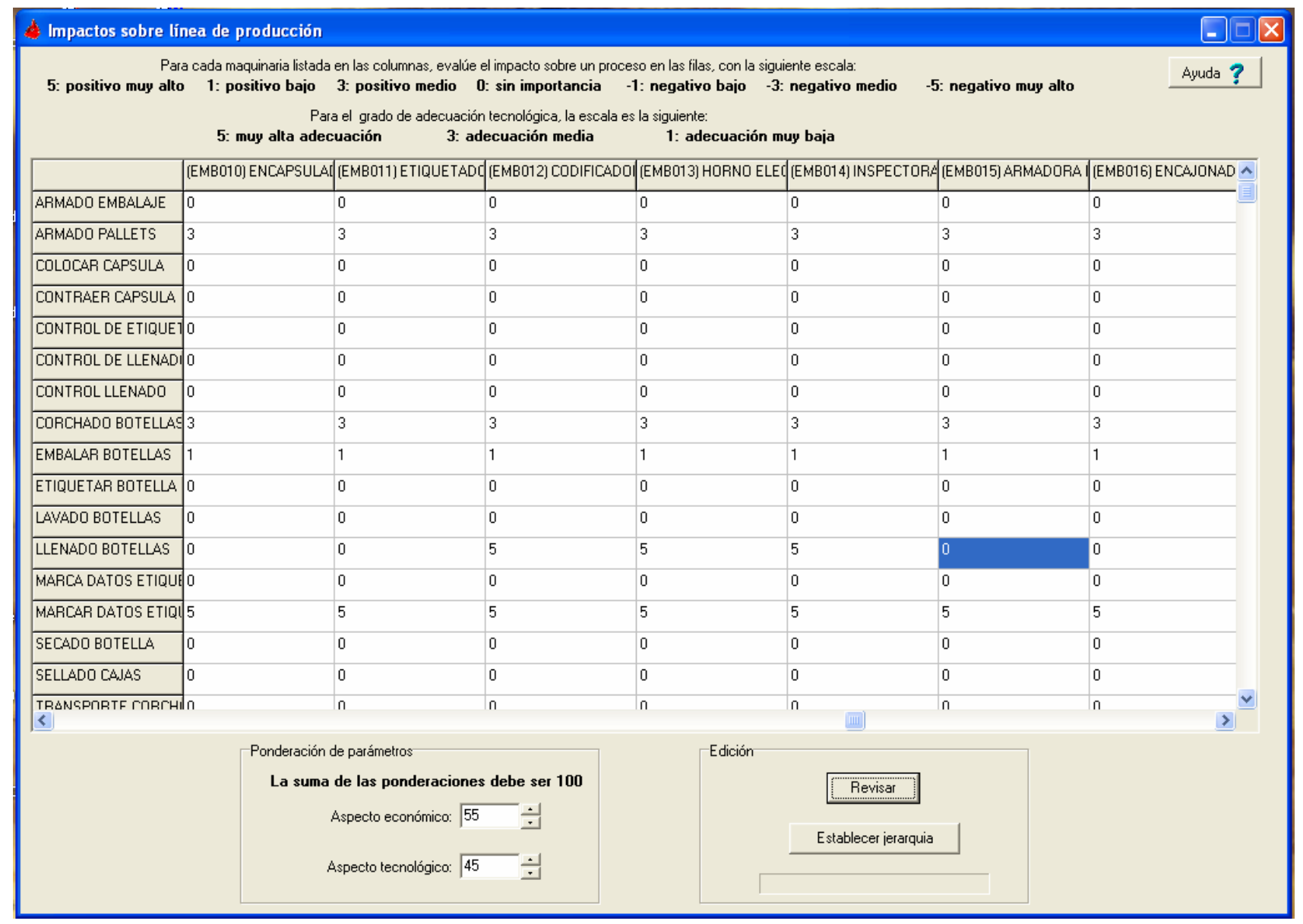

Fig. 5 Pantalla de captura de datos de relaciones de máquinas y procesos productivos

La matriz para el análisis de los resultados está compuesta de dos grupos de indicadores: un grupo referido a la situación actual del equipamiento y al impacto de la innovación en la línea de producción y el otro grupo al costo de adquisición del equipamiento y a la criticidad del equipamiento. La primera lista ordenada entregada por la matriz está referida a la situación actual del nivel de atendimiento de los equipamientos en relación a las metas impuestas para obtener un producto, de acuerdo con la calidad que el mercado exige.

El primer criterio - $C r_{1, j}$ - (Media del atendimiento / Impacto $(M / I)$ ) es calculado por la razón entre el nivel de atendimiento del equipamiento j considerado - MAj - (Media del atendimiento por equipamiento) e - $I P_{i, j}$ - (Impacto del equipamiento en el proceso) el impacto total en la línea de producción del equipamiento j en los procesos de fabricación $i$ (Fig. 6).

Para cada equipo $j$ :

$C r_{1, j}=\frac{M A_{j}}{\sum_{i=1}^{n} I P_{i, j}} \quad i=1,2, \ldots n$ processos

La máquina asociada al valor más bajo debería tener prioridad en la adquisición, ya que esto indica que el equipamiento posee un nivel bajo de atendimiento de los requisitos del negocio de la empresa y, además de eso, un alto impacto en los procesos de fabricación de la línea de producción.

El segundo criterio - $C r_{2, j}$ - (Valor de la inversión / Criticidad (V/C)) es calculado mediante la razón entre la criticidad del equipamiento j - CRIT - (Criticidad del equipamiento de la línea) y el costo de adquisición de este $-C E_{j}-($ Valor de la inversión (US\$)), cuyo valor es introducido por el analista (Ver Fig. 6). 
Jerarquización de inversión en el parque de maquinarias

Jerarquización de inversión en el parque de maquinarias

\begin{tabular}{|c|c|c|c|c|c|c|c|c|c|}
\hline \begin{tabular}{|l|} 
Jerarquia \\
\end{tabular} & Maquinaria & Indice & Criticidad & Impacto total & Media atendimiento & Valor adquisicion/Criticidad & Jerarquia $V / C$ & Media atend./Impacto & Jerarquia $M, \wedge$ \\
\hline 1 & (EMB008) INSPECTOR DE NIVEL M & 100.00 & 40.0 & 17 & 1.77 & 2.1250 & 2 & 0.1043 & 1 \\
\hline 2 & (EMB014) INSPECTORA DE ETIQUI & 108.49 & 62.5 & 17 & 2.41 & 0.9600 & 1 & 0.1417 & 8 \\
\hline 3 & (EMB013) HORNO ELECTRICO & 132.03 & 82.5 & 17 & 1.91 & 4.0000 & 6 & 0.1123 & 3 \\
\hline 4 & (EMB012) CODIFICADORA WILLET & 156.35 & 52.5 & 17 & 2.05 & 5.3333 & 9 & 0.1203 & 4 \\
\hline 5 & (EMB005) CORCHADORA KHS & 172.52 & 40.0 & 12 & 1.50 & 6.2500 & 11 & 0.1250 & 5 \\
\hline 6 & (EMB007) CODIFICADORA WILLET & 179.34 & 47.5 & 17 & 1.82 & 7.5789 & 12 & 0.1070 & 2 \\
\hline 7 & (EMB006) ELEVADOR DE CORCHO & 187.37 & 22.5 & 12 & 2.00 & 5.3333 & 9 & 0.1667 & 9 \\
\hline 8 & (EMB010) ENCAPSULADORA BEEF & $=188.03$ & 75.0 & 12 & 2.18 & 4.6667 & 8 & 0.1818 & 11 \\
\hline 9 & (EMB011) ETIQUETADORA KRONE & $=191.05$ & 52.5 & 12 & 2.45 & 3.8095 & 5 & 0.2045 & 14 \\
\hline 10 & (EMB016) ENCAJONADORA & 191.56 & 60.0 & 12 & 2.50 & 3.6667 & 4 & 0.2083 & 15 \\
\hline 11 & (EMB018) SELLADORA & 197.62 & 60.0 & 12 & 2.86 & 2.6667 & 3 & 0.2386 & 16 \\
\hline$\overline{12}$ & (EMB004) LLENADORA KHS & 215.10 & 75.0 & 12 & 1.55 & 9.0667 & 13 & 0.1288 & 6 \\
\hline 13 & (EMB017) BALANZA & 241.92 & 75.0 & 12 & 3.32 & 4.0000 & 6 & 0.2765 & 19 \\
\hline 14 & (EMB009) SECADOR HESS & 251.35 & 60.0 & 12 & 2.00 & 9.8333 & 14 & 0.1667 & 9 \\
\hline 15 & (EMB003) ENJUAGADORA KHS & 326.88 & 52.5 & 12 & 1.64 & 16.5714 & 16 & 0.1364 & 7 \\
\hline 16 & (EMB015) ARMADORA DE CAJA & 391.13 & 32.5 & 12 & 3.09 & 15.3846 & 15 & 0.2576 & 17 \\
\hline 17 & (EMB002) CINTA TRANSPORTADO & 448.69 & 70.0 & 12 & 2.36 & 22.2857 & 17 & 0.1970 & 13 \\
\hline 18 & (EMB019) ROBOT PALLETIZADOR & 594.53 & 75.0 & 12 & 3.18 & 29.3333 & 18 & 0.2652 & 18 \\
\hline
\end{tabular}

Para exportar éste resultado aXMLSS, hacer click con el botón derecho del mouse sobre la tabla.

La jerarquización fue realizada en base a la poderación del aspecto económico (55\%) y el aspecto tecnológico ( $45 \%$ ).

La máquina que aparece en primer lugar debería ser considerada para una evaluación de proyectos teniendo en mente

las alternativas de reemplazarla por una nueva o bien modernizar su nivel tecnológico.

Fig. 6 Matriz de jerarquización para definir prioridades de inversión

\section{Para cada equipo $j$}

$C r_{2, j}=\frac{C E_{j}}{C R I T_{j}}$

Este indicador da preferencia a aquel equipamiento cuya criticidad es alta y su costo de adquisición es bajo, o sea, lleva en cuenta no solamente el aspecto económico, sino también su importancia en la confiabilidad de la línea de producción. Debería tener prioridad el equipamiento asociado al indicador con mayor valor.

El tercer indicador mostrado en la Fig. 6 es la conjunción de los dos indicadores anteriormente nombrados, y aquel equipamiento con el ordenamiento en los primeros lugares de las listas anteriores, quedará con la más alta preferencia para ser introducido en la línea de producción, ya que es lo que mejor atiende todas las condicionantes para una buena selección.

En la matriz se hace referencia a una variable llamada de grado de adecuación a la tecnología, que puede ser una condicionante que cambie todas las prioridades definidas por el modelo y esta variable es de difícil modelamiento por ser muy subjetiva. La variable, grado de adecuación puede influir en la redefinición de la tecnología del equipamiento, o en el atraso de la introducción de la máquina, debido a exigencias de capacitación de los operadores y mantenedores, obras en infraestructura entre otras restricciones.

Otro dato que se muestra en la primera columna de la Fig.6 es el parámetro denominado "Índice" y se usa para mostrar cuán fuerte es el impacto de un equipo en referencia al primero de la lista jerarquizada, así por ejemplo, la jerarquía 1 con respecto a la 18, es seis veces más impactante. Todos los datos que se muestran en la Fig. 6 pueden ser exportados a una planilla Excel y definir otros indicadores o cálculos que el analista estime pertinente para la toma de decisiones. 


\section{CONCLUSIÓN}

Se debe resaltar que este modelo es solamente un procedimiento de ayuda para el administrador y su equipo de personas con la finalidad de darles una visión holística del problema atendiendo variables que se relacionan con una análisis del escenario que deberá enfrentar el mantenimiento para atender los cambios de las condicionantes que imponen la evolución del producto en el mercado, y de la situación actual, que es resultado de introducir un equipo productivo nuevo en la línea de producción. Las variables que maneja el modelo describen de buena forma el proceso de toma de decisiones de los involucrados en mantener la eficiencia de una línea de producción, tal como fue comprobado en el proceso de validación del modelo, en donde además se constató una externalidad positiva: obligó a los mantenedores a involucrase en el desarrollo y evolución del negocio de la empresa.

Pero pueden existir condicionantes especiales que harán con que la sugerencia entregada por el modelo quede sin validez, como ser la urgencia en la adquisición del equipamiento o un presupuesto muy pequeño. Entonces el administrador tendrá que decidir sobre la adquisición del equipamiento solamente por un único factor que es el costo de pérdida de ventas o costo del equipamiento. También puede acontecer que la influencia de la introducción del equipamiento en la línea de producción sea casi nula para el resto de los procesos y en este caso no es necesario realizar un análisis de este tipo, ya que el problema está restringido a un solo punto en la línea de producción, o que la condición del nivel de bajo atendimiento de los requisitos se concentre solamente en pocos equipamientos para lo cual la solución puede ser directa.

\section{REFERENCIAS}

Bhuiyan N. y Baghel A. An overview of continuous improvement: from the past to the present. Management Decision, Vol. 43 No. 5, 761-771 (2005).

Carneiro A. Adopting new technologies. Handbook of Business Strategy. 307-312, Emerald Group Publishing Limited, ISSN 0894-4318 (2006).

Chan F.T.S., Lau H.C.W., Ip R.W.L., Chan H.K., Kong S. Implementation of total productive maintenance: a case study. International Journal of production Economics. Volume 95, Issue 1, 71-94 (2005).

Chen I., Small M. Planning for advanced manufacturing technology. International Journal Of Operation \& Production Management. Vol. 16, No. 5. 4-24 (1996).

Coetzee J.L. A holistic approach to the maintenance "problem". Journal of Quality in Maintenance Engineering, Vol. 5 No. 3, 276-280 (1999).

Crespo A., Moreu de León P, Gómez J.F., Parra C.y López M. The maintenance management framework: practical view to maintenance management. Journal of Quality in Maintenance Engineering. Vol. 15 No. 2, 167-178 (2009).

Kumar R., Singh S. R., y Markeset T. Development of technical integrity management services - a concept. Journal of Quality in Maintenance Engineering Vol. 15 No. 3, 271-284 (2009).

Rentes A. Transmeth - Proposta de uma Metodologia para Condução de Processos de Transformação de Empresas. Universidade de São Paulo Escola de Engenharia de São Carlos (2000).

Riis J.O., Luxhoj J.T., Thorsteinsson U. A situational maintenance model. International Journal of Quality \& Reliability Management. Vol 14 No 4. pp. 349-366 (1997).

Shyjith K., M. Ilangkumaran y S. Kumanan. Multi-criteria decision-making approach to evaluate optimum maintenance strategy in textile industry. Journal of Quality in Maintenance Engineering, Vol. 14 No. 4, 375 - 386 (2008).

Suárez D.J., Espinoza H.J., Collado M.L., Rodríguez E.A., Metodología de clasificación de equipos según su criticidad utilizando los criterios del mantenimiento centrado en la confiabilidad. Información Tecnológica, Vol. 14(2), 143-148 (2003). 